\section{hommes}

\section{Hommes \& migrations}

Revue française de référence sur les dynamiques

migratoires

1312 | 2015

Diasporas iraniennes

\title{
Des Iraniens à Erevan : un « cosmopolitisme vernaculaire »?
}

\section{Amin Moghadam}

\section{(apenEdition \\ Journals}

\section{Édition électronique}

URL : http://journals.openedition.org/hommesmigrations/3489

DOI : 10.4000/hommesmigrations.3489

ISSN : 2262-3353

\section{Éditeur}

Musée national de l'histoire de l'immigration

\section{Édition imprimée}

Date de publication : 1 octobre 2015

Pagination : 33-41

ISBN : 978-2-919040-33-9

ISSN : $1142-852 X$

\section{Référence électronique}

Amin Moghadam, « Des Iraniens à Erevan : un « cosmopolitisme vernaculaire »? », Hommes \& migrations [En ligne], 1312 | 2015, mis en ligne le 01 octobre 2018, consulté le 22 avril 2019. URL : http://journals.openedition.org/hommesmigrations/3489; DOI : 10.4000/hommesmigrations.3489 


\title{
DES IRANIENS À EREVAN : UN "COSMOPOLITISME VERNACULAIRE" ?
}

par AMIN MOGHADAM, université Aix-Marseille, chercheur associé à l'Urmis, et SERGE WEBER, université Paris-Est Marne-la-Vallée, laboratoire Analyse comparée des pouvoirs.

\author{
Dans un contexte où les sanctions internationales ont \\ recomposé les mécanismes de fonctionnement de l'économie \\ iranienne, la République islamique d'Iran a fortement développé \\ son rayonnement régional. La République d'Arménie toute \\ proche est devenue un carrefour de circulations migratoires, \\ commerciales et culturelles avec I'Iran. Une enquête \\ à Erevan au printemps 2015 permet de saisir la diversité et \\ l'impact de ces échanges sur l'économie et la culture \\ iraniennes. Une manière, pour les Iraniens, d'expérimenter \\ le cosmopolitisme, de l'autre côté de la frontière.
}

Pour les nouvelles classes moyennes iraniennes qui ont émergé après la révolution de 1979, les espaces de l'étranger proche sont devenus des lieux ressources pour accéder à des formes de socialisation à l'international ${ }^{1}$. À la différence de certains de leurs compatriotes qui disposent de liens à une échelle globale de la diaspora, que ce soit en Europe occidentale, aux États-Unis ou plus récemment en Australie, ces Iraniens trouvent dans des villes comme Istanbul, Dubaï, Erbil (au Kurdistan irakien), Tbilissi ou Erevan, des opportunités moins inaccessibles de voyager. Elles leur permettent de faire face aux effets indirects des sanctions internationales adoptées par la plupart des pays industrialisés à l'égard de l'Iran depuis 2000 et surtout depuis 2010. Ces effets indirects sont aussi bien les restrictions draconiennes de l'accès aux visas pour circuler vers les pays occidentaux que les entraves aux transactions commerciales du fait de l'exclusion de l'Iran du système des règlements financiers internationaux.

Par ailleurs, l'Iran a concentré ses efforts diplomatiques sur l'échelle régionale, à défaut de pouvoir développer des réseaux de commerce à l'échelle internationale et globale, dans une politique que l'on peut qualifier de régionaliste ${ }^{2}$. Cette politique s'appuie sur différents acteurs, entrepreneurs, voyageurs, touristes, qui deviennent les instruments des relations économiques avec l'étranger, par le haut et par le bas, formelles et informelles ${ }^{3}$. 
Cet article se concentre sur les voyages, les circulations et les lieux de sociabilité des Iraniens à Erevan, capitale de la République d'Arménie. Les échelles nationales et transnationales sont particulièrement imbriquées avec l'histoire des déplacements des populations et des minorités dans la région.

\section{Iran-Arménie : des liens anciens et réactivés}

Les relations migratoires entre l'Iran et l'Arménie sont étroitement liées à la période où le territoire de l'Arménie actuelle faisait partie de l'Empire perse - la mosquée chiite d'Erevan a été construite au XVIII siècle - et à l'ancienneté des populations arméniennes en Iran. Initialement situées dans le nord du pays (notamment à Djolfa), les minorités arméniennes ont été déplacées par le Shah Abbas dans la région d'Ispahan au XVIe siècle. Depuis, les Arméniens d'Iran

se sont installés dans d'autres villes du pays, ainsi qu'à Téhéran. Durant le génocide de 1915, beaucoup d'Arméniens d'Anatolie ont trouvé protection dans la monarchie qâdjâr : ils constituent avant la révolution une minorité nombreuse d'environ 200000 personnes $^{4}$. Après la La circulation a ainsi révolution de 1979, environ changé de statut : elle s'est reportée sur les voyages de tourisme, avec 90 ooo visiteurs iraniens en Arménie en 2014

c'est donc aujourd'hui une mobilité à court terme et renouvelée. 50000 Iraniens arméniens ont quitté le pays 5 , notamment vers l'Europe, les ÉtatsUnis, l'Inde ou l'Asie du SudEst. Certains ont choisi l'URSS, en particulier la République socialiste soviétique d'Arménie. Parmi ces derniers, beaucoup ont fait leurs études à Moscou ou à Leningrad avant de s'installer à Erevan. Au moment de l'effondrement de l'URSS, les frontaliers ont commencé à faire du commerce entre l'Arménie et le
La mosquée chiite d'Erevan.

(c) Serge Weber.

nord-ouest de l'Iran. Ce sont des commerçants de la minorité azérie d'Iran qui ont initié les échanges, d'où la présence précoce d'une importante communauté commerçante azérie d'Iran à Erevan, originaire notamment de Tabriz (capitale économique de l'Azerbaïdjan iranien). Ces commerçants tiennent encore la majorité des quelques restaurants iraniens de la capitale.

La phase suivante, de la fin des années 1990 au début des années 2010, est caractérisée par une sorte d'"âge d'or", durant lequel les Iraniens non arméniens vont nombreux à Erevan : beaucoup d'étudiants viennent faire leurs études à l'université d'État d'Erevan ou dans les universités étrangères de la ville (université américaine, université française), notamment en médecine, agronomie, architecture, ingénierie et histoire de l’art. 
On comptait alors environ 3000 étudiants et 300 commerçants installés.

Mais, durant la courte période allant de 2010 à 2013, la Géorgie devient un pôle concurrent. Le nombre de résidents iraniens serait passé de 10000 à 3 000. Depuis, le déclin est très net. En 2014, on compte au maximum entre 700 et 800 étudiants iraniens à Erevan, pour une raison très simple : l'Iran a cessé de reconnaître l'équivalence des diplômes dans la plupart des disciplines, exception faite de certaines filières comme l'architecture et l'histoire de l'art, pour avoir découvert que beaucoup d'étudiants inscrits ne fréquentaient pas l'université et qu'il était possible d'acheter certains diplômes. La plupart des commerçants ont quitté le pays. Les marchés alimentaires sont désertés par les commerçants iraniens, de même que le marché de gros situé en périphérie, où il ne reste qu'un restaurant iranien pour les quelques transporteurs qui continuent à faire de l'import-export. La circulation a ainsi changé de statut : elle s'est reportée sur les voyages de tourisme, avec 90000 visiteurs iraniens en Arménie en 2014, c'est donc aujourd'hui une mobilité à court terme et renouvelée. Parmi eux, un certain nombre de "touristes" viennent en Arménie afin d'obtenir un visa pour les États-Unis, depuis la désignation du consulat américain d'Erevan pour le traitement des demandes iraniennes.

\section{Voyager, s'installer : des contraintes maîtrisées}

Depuis les années 1990, les accords mutuels entre l'Arménie et l'Iran facilitent l'obtention du visa, à l'aéroport et à la frontière terrestre. Ces mêmes mesures ont été appliquées aux Arméniens qui se rendent en Iran, grâce au développement des services consulaires sur le territoire arménien, notamment au point de frontière terrestre de Nordouz. Un dialogue est en cours sur la suppression réciproque des visas.
L'accès à un éventail plus large de droits est conditionné par l'obtention de la citoyenneté ou d'un statut équivalent. Celle-ci est différenciée, sinon de jure, au moins de facto, en fonction de l'ethnicité : les Iraniens de la minorité arménienne font l'objet de traitements spécifiques en Arménie par rapport aux autres. Néanmoins, l'accès à la citoyenneté ne va pas sans poser de problème, notamment pour les jeunes hommes soumis à l'obligation de service militaire dans les deux pays. La conservation de la double nationalité étant

tolérée par les deux gouver- Malgré la proximité, nements, c'est une solution les conditions de transport majoritairement adoptée, restent un obstacle à associée à des allers-retours la fluidité des échanges. réguliers. Pour les Iraniens Si les liaisons aériennes sont qui ne sont pas issus de la devenues plus régulières, minorité arménienne, l'accès le réseau routier n'est pas à la nationalité arménienne toujours adapté aux flux. est restreint. Il est possible d'obtenir une carte de résidence de cinq ans ouverte de droits sous deux conditions : après avoir renouvelé trois fois un permis d'un an ou à la suite d'un mariage avec un(e) ressortissant(e) arménien(ne) dans la plupart des cas, il s'agit d'un mariage entre un homme iranien et une femme arménienne. Cette carte de séjour de cinq ans donne accès à de nombreux droits des nationaux, notamment le droit à la santé et l'accès à l'hôpital public aux prix nationaux. Malgré la proximité, les conditions de transport restent un obstacle à la fluidité des échanges. Si les liaisons aériennes sont devenues plus régulières, le réseau routier n'est pas toujours adapté aux flux. Les liaisons par autocar sont régulières, mais les investissements font défaut : la route menant en Iran est en mauvais état (il faut quatorze heures pour parcourir en car les $520 \mathrm{~km}$ séparant Erevan de Tabriz). Selon des diplomates iraniens, des négociations sont en cours pour des travaux de modernisation des routes et la construction d'une liaison ferroviaire qui prolongerait jusquau Caucase l'hinterland du port iranien de Bandar Abbas sur le golfe Persique. 


\section{Le développement des échanges culturels}

La présence des Iraniens à Erevan et l'intensification des échanges alimentent un "secteur iranien" offrant des services correspondant à une demande multiforme : d'une part, des formes institutionnelles, d'autre part, des pratiques moins visibles. L'enseignement supérieur est un bon observatoire des échanges culturels qui consolident le champ migratoire. Le département d'études iraniennes dans la faculté d'études orientales de l'université d'État d'Erevan est un centre réputé dans la géographie mondiale de l'iranologie, au point d'attirer des étudiants de divers pays (États-Unis et Russie principalement) et d'inviter des spécialistes étrangers, européens ou américains, pour des conférences. C'est là qu'est éditée la revue Iran and the Caucasus, reférence aca-

Parallèlement, le service culturel de l'ambassade d'Iran poursuit une initiative ambitieuse dans l'enseignement secondaire arménien, en plus de l'enseignement en persan de l'école iranienne située rue Abovyan, qui va de la maternelle au bac. démique publiée à Leyden (Pays-Bas). Environ 200 étudiants y étudient le persan et l'iranologie.

Parmi les diplômés, certains vont compléter leurs études par des séjours universitaires en Iran, même s'il n'existe pas d'accord formel d'échange interuniversitaire. Dans notre enquête, c'est la fondation Saadi de l'université internationale Khomeiny de Qazvin et l'université Shaid Beheshti de Téhéran qui sont apparues comme des destinations privilégiées, deux pôles universitaires renommés. Trouver un emploi à Erevan avec cette compétence linguistique s'avère aisé, au vu des coopérations diplomatiques, de l'activité touristique iranienne florissante et de la demande en cours de russe par des Iraniens qui souhaitent émigrer, notamment pour des études supérieures, en Russie. Il y a donc ce qu'on pourrait appeler un secteur iranien, qui est un débou- ché professionnel pour les étudiants arméniens. "Comme job, on est guide touristique pour les Iraniens pendant les congés de Norouz et l'été. On apprend le persan littéraire. La première fois que mon chef à l'agence m'a demandé de faire une visite guidée en persan, c'était difficile car je parlais littéraire aux Iraniens, avec des tournures poétiques, mais, au bout d'une journée, je me suis faite au registre de la langue du quotidien sans problème" (entretien avec deux étudiantes arméniennes, Erevan, 2015).

Parallèlement, le service culturel de l'ambassade d'Iran poursuit une initiative ambitieuse dans l'enseignement secondaire arménien, en plus de l'enseignement en persan de l'école iranienne située rue Abovyan, qui va de la maternelle au bac.

"Dans le cadre des accords culturels signés entre les deux pays, nous avons institué l'enseignement du persan dans dix écoles arméniennes dès le collège et le lycée, au choix comme langue étrangère au même titre que l'anglais ou le français. Quand ils arrivent au bac, ils ont déjà une connaissance du persan et peuvent poursuivre leurs études au département d'études iraniennes. L'Iran a aidé quelques-unes de ces écoles pilotes à moderniser leurs équipements, à digitaliser leurs enseignements, à installer des tableaux numériques. Il y aurait aujourd'hui 800 à 900 élèves de collèges et lycées dans les écoles arméniennes qui apprennent le persan. Un des meilleurs exemples, c'est l'école 'numéro 200' de la ville d'Erevan, modernisée à l'aide du gouvernement iranien" (entretien avec un diplomate iranien, Erevan, 2015).

Enfin, la mosquée iranienne d'Erevan n'est pas qu'un symbole national en dehors des frontières, c'est aussi un lieu où les migrants iraniens peuvent suivre des cours d'arménien et même de russe pour ceux qui préparent une émigration vers la Russie.

\section{Un carrefour artistique iranien}

Les circulations artistiques et l'émergence de marchés de l'art sont des indicateurs de l'intégration régionale et de l'émergence de polarités et de 
réseaux. À Erevan, on remarque l'émergence, certes timide, d'un marché de l'art. C'est essentiellement du fait de l'implication active de certains entrepreneurs culturels conscients de trois défis à relever. Le premier est l'enjeu national et identitaire du public et des financeurs locaux, dont le projet culturel est d'ancrer l'affirmation politique des Arméniens en train de faire leurs comptes avec l'histoire de l'oppression. Le deuxième enjeu est la concurrence forte des grands pôles comme Dubaï, Istanbul et Beyrouth, qui dominent clairement le marché régional de l'art. Le troisième est la relative étroitesse de la clientèle locale potentielle, à la fois peu nombreuse et pas forcément familière de l'avant-garde. C'est donc à une équation délicate que ces entrepreneurs culturels sont confrontés. Cependant, ils s'avèrent des acteurs clés de l'émergence de champs transnationaux de circulation de modèles culturels globalisés, dans l'expression d'une singularité régionale par rapport aux marchés dominants européens, nord-américains et est-asiatiques. De plus, les artistes sont nombreux et actifs, même s'ils peinent à se connecter à des réseaux de distribution et de promotion.

Nous présentons ici le cas de figure d'une galerie d'Erevan dirigée par une Arménienne originaire d'Iran, dont le point de vue est particulièrement intéressant sur le rapport aux artistes et galeristes d'Iran et aux autres marchés de l'art en diaspora. "Cet espace pourrait devenir un lieu pour la diaspora arménienne, d'ailleurs ou d'Iran, et des Iraniens de la diaspora qui se retrouveraient ici pour différents types d'activité. (...) Notre clientèle est composée de beaucoup d'Arméniens d'Iran qui habitent ici et sont intéressés par l'art et la littérature" (entretien avec une galeriste, Erevan, 2015).

Les Arméniens d'Iran émigrés participent à la diffusion des productions culturelles iraniennes: "On va projeter Le Passé, on a mis une annonce sur notre page Facebook et beaucoup d'Arméniens iraniens et
d'Iraniens d'ici voulaient venir à la projection du film, en français doublé en persan, et on a trouvé de l'argent pour les sous-titres en arménien. On travaille avec un ami régisseur arménien iranien qui habite en France, il choisit des films et nous, avec des fonds de la galerie, on finance le sous-titrage" (ibid.).

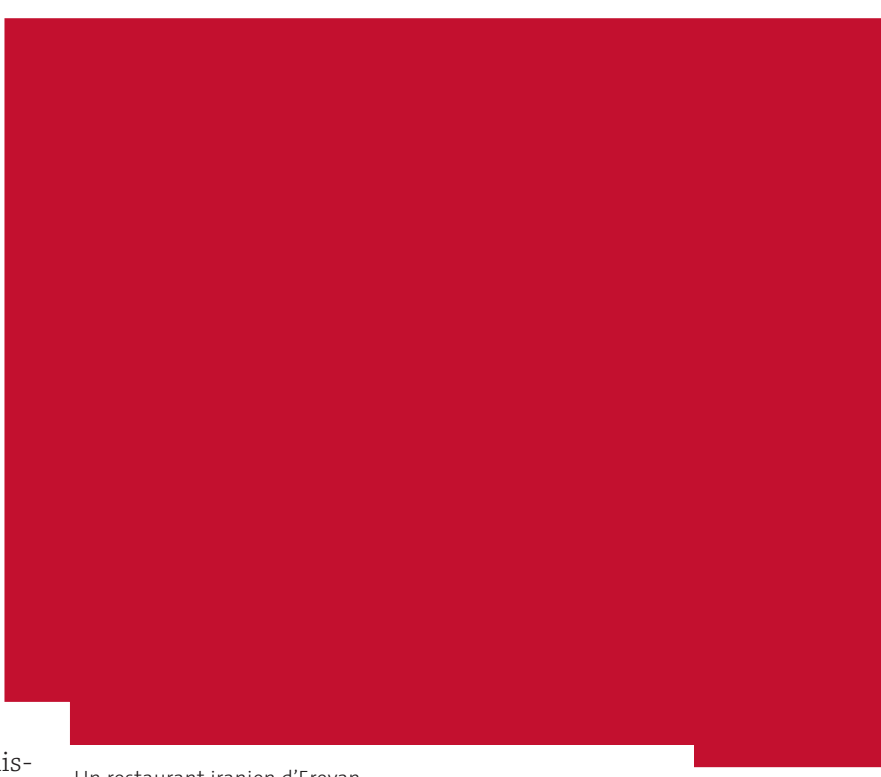

Un restaurant iranien d'Erevan. (c) SERGE Weber.

En parallèle, l'Arménie est un lieu de tournage pour beaucoup de réalisateurs iraniens, qui peuvent ainsi réduire les coûts de production et qui constituent une clientèle pour les quelques sociétés de production de la capitale.

Erevan est aussi un refuge pour les musiciens exilés, à l'exemple de ce jeune auteur compositeur, guitariste et bassiste de 32 ans qui, après s'être formé au jazz et au rock à Téhéran, a connu une relative notoriété dans divers styles (rock, trans et pop) et a réalisé un album avec un chanteur assez connu, mais il a eu des problèmes avec la censure à propos de son style. Il a donc jugé plus prudent de quitter le pays et de s'installer à Erevan, en 20146. 


\section{Erevan, une destination touristique de choix}

Erevan se présente donc pour les Iraniens comme un "espace par procuration" ou une "zone franche morale", dans l'esprit des travaux de Franck Mermier ${ }^{7}$, pour certaines formes de tourisme permettant d'échapper quelques jours aux interdits régissant la vie sociale en Iran.

Les visiteurs iraniens en Arménie constituent une clientèle pour de nombreuses agences : une véritable économie du tourisme iranien s'est mise en place, encadrée par des Iraniens d'Erevan et des Arméniens qui parlent le persan, qu'ils soient originaires d'Iran ou qu'ils aient appris le persan comme langue étrangère. De nombreux Iraniens viennent en touristes pour quelques jours à Erevan. Les pics de fré-

De nombreux Iraniens viennent en touristes pour quelques jours à Erevan.

Les pics de fréquentation sont les fêtes du nouvel an

de Norouz et les congés d'été. Pour eux, il s'agit d'un tourisme de consommation en réaction aux interdits imposés le reste du temps. quentation sont les fêtes du nouvel an de Norouz et les congés d'été. Pour eux, il s'agit d'un tourisme de consommation en réaction aux interdits imposés le reste du temps. La consommation d'alcool, la fréquentation de "clubs" (boittes de nuit), la rencontre de "filles" (prostituées, entraîneuses ou simples partenaires de danse) et la consommation sexuelle constituent certains des buts du voyage. Il y aurait dans la ville au moins cinq boîtes de nuit où les hôtesses parlent persan. Nous avons rencontré un agent informel de location d'appartements appartenant à des Arméniens pour touristes iraniens. Il se présente lui-même comme un "atchar faransê" ("tournevis français"), en d'autres termes un débrouillard qui maitrise un large éventail d'activités, de ressources et d'informations au service des visiteurs iraniens. Cet exemple est significatif du développement d'un nouveau

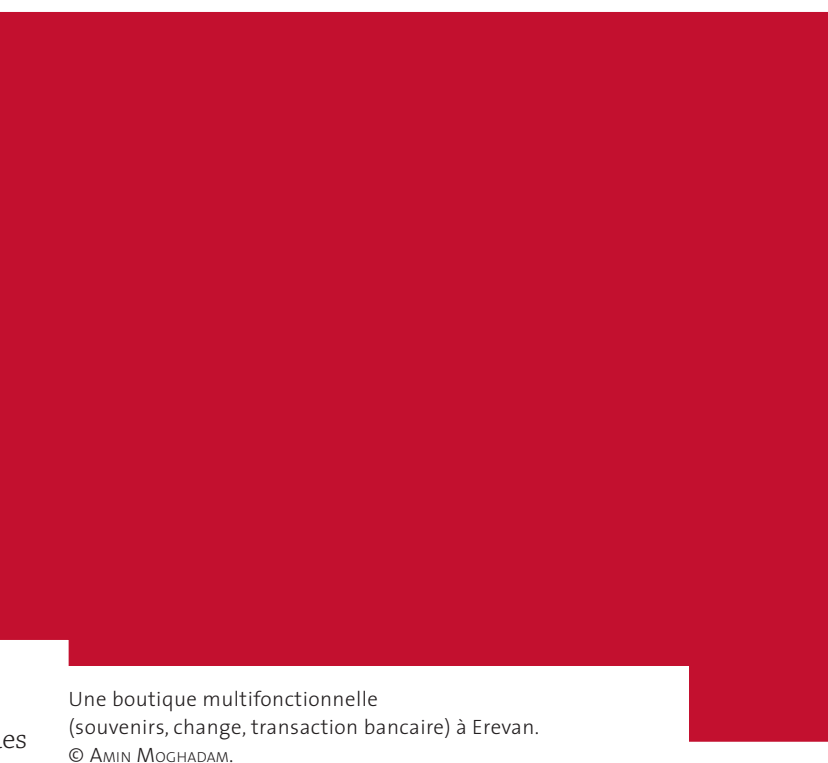
(c) Amin Moghadam.

type de marketing : étant donné l'existence d'une clientèle, des entrepreneurs ont choisi d'en faire un business, notamment dans les lieux de passage des voyageurs iraniens, à proximité du terminal d'autocar situé dans le centre-ville ou sur la route jusquà la frontière.

La place de la République est un lieu où des "rabatteurs" cherchent des clients iraniens pour leur proposer divers services : des visas pour des pays occidentaux (non-UE), des sorties dans des clubs et restaurants iraniens (il suffit de faire une réservation, ils envoient le taxi chercher les clients, qui les conduit dans les clubs), des procédures administratives pour travailler, investir ou acheter une propriété en Arménie. Ces agences de tourisme, qui ont des représentations à Téhéran, servent même de banques informelles par procuration. Recommandées parfois par l'agence bancaire iranienne officielle aux visiteurs à court d'argent, elles fournissent de l'argent liquide en échange d'un numéro de carte bancaire iranienne - exclue du système bancaire international.

On croise des Iraniens de tous âges, depuis les étudiants jusqu'aux retraités : le va-et-vient donne aux espaces publics centraux, pour qui sait dres- 
ser l'oreille ou interpréter les codes vestimentaires, une connotation discrètement persane. Les restaurants iraniens sont des étapes obligées des touristes, ils emploient des serveurs et serveuses arméniens qui ont appris le persan pour accueillir les clients, quelle que soit la raison de leur présence à Erevan.

\section{Le commerce formel et informel au service d'une même économie}

L'impossibilité juridique pour les Iraniens d'accéder à l'Union européenne et à ses partenaires d'association économique (politique de voisinage, unions de libre-échange), comme la Géorgie, contraint les mobilités internationales professionnelles (commerce, import-export) à inventer de nouvelles destinations, de nouveaux projets, à rechercher de nouveaux liens-ressources. L'alternative permanente reflète l'obligation que l'Iran a de reconfigurer ses espaces de commerce international en fonction des entraves imposées par les sanctions. Plus encore, du fait des sanctions, aucune entreprise iranienne, privée ou publique, n'a accès au marché international des changes, ce qui les prive d'accès au règlement monétaire des transactions garanti par le réseau SWIFT ${ }^{8}$.

Le transfert informel d'argent (hawaleh en persan, qu'on retrouve dans tout le Moyen-Orient, jusqu'à l'Inde avec le hundi), constitue donc la seule manière de procéder à des échanges financiers. Le rôle de la diaspora et des Iraniens qui détiennent des comptes à l'étranger est donc accru pour ces intermédiaires indispensables - d'où l'importance de l'historicité de la présence des marchands persans au-delà des frontières ${ }^{9}$. L'import-export de pièces détachées pour automobiles comme de médicaments, deux secteurs théoriquement exemptés des sanctions depuis l'accord préliminaire passé à Genève en novembre 2013, souffre précisément de ce problème : aucune compagnie n'accepte de vendre ces produits, sachant que, même hors sanction, ils ne seront pas payables. C'est d'ailleurs pour cette raison qu'un certain nombre d'échanges, notamment avec l'Arménie, ont été faits sous forme de troc. "On n'a pas accès aux transactions bancaires sur le SWIFT. Quand on vend ici, on ne peut pas envoyer l'argent en Iran, on est obligé de passer par un bureau de change. Il faut les connaître, ils doivent connaître quelqu'un en Iran. Si on achète un produit en Arménie, il faut payer quelqu'un: c'est un transfert informel d'argent par les bureaux de change. L'acheteur ou le vendeur doit donc payer une commission à ces intermédiaires des bureaux de change" (entretien avec un concessionnaire automobile iranien, Erevan, 2015). C'est donc bien là que réside la clef de voûte de cette dépendance mutuelle du formel et de l'informel : pour qu'il y ait des échanges formels, il faut en passer par un marché des changes informel, reposant sur l'interconnaissance et les complaisances administratives $^{10}$.

L'Iran a vu sa position commerciale avec l'Arménie diminuer ces dernières années :
L’impossibilité juridique pour les Iraniens d'accéder à l'Union européenne et à ses partenaires d'association économique (politique de voisinage, unions de libre-échange), comme la Géorgie, contraint les mobilités internationales professionnelles (commerce, import-export) à inventer de nouvelles destinations, de nouveaux projets, à rechercher de nouveaux liens-ressources. partenaire de l'Arménie, après avoir été le deuxième au cours des années 2000. L'accord d'ouverture commerciale de la Géorgie avec l'Union européenne a conduit l'Iran à restreindre les échanges avec son partenaire principal, l'Arménie. De ce fait, étant exclue de l'union entre la Géorgie et l'UE, l'Arménie a rejoint l'Espace économique eurasiatique (EEU, Eurasian Economic Union, associant Russie, Biélorussie, Kazakhstan et Kirghizstan), qui lui permet d'avoir depuis janvier 2015 une circulation libre de la main-d'œuvre et des produits 
dans ces pays. Mais cela a aussi encouragé l'Arménie à avoir davantage de relations avec l'Iran.

L'Iran s'impose donc malgré tout comme un partenaire commercial pour l'Arménie, exportant du gaz et une gamme restreinte de produits de consommation courante (notamment ménagers). Cette présence commerciale iranienne se lit dans un contexte politique particulier. D'un côté, même si l'Iran n'a guère besoin de l'électricité arménienne, elle choisit d'en importer pour renforcer le partenariat et exporter du gaz. Cela sous l'œil méfiant de la Russie, rappelant que la position de l'Arménie est bien fragile dans cette intrication d'accords qui confirment sa situation de dépendance. L'Iran conquiert donc des niches secondaires. La réciproque est vraie, puisque la principale exportation arménienne vers l'Iran est constituée d'ovins et de caprins vivants. "Il y a beaucoup d'exportateurs arméniens de moutons et de chèvres vers l'Iran. Allez dans les supermarchés : certains produits L'Iran s'impose donc de consommation courante (shammalgré tout comme pooing) sont de marque iranienne.

un partenaire commercial pour l'Arménie, exportant

du gaz et une gamme

restreinte de produits

de consommation courante

(notamment ménagers).
De toute manière, l'Arménie est un petit marché de 2 millions d'habitants, ce rest pas un gros marché" (entretien avec un entrepreneur iranien à Erevan, 2015).

En effet, par sa taille modeste, l'Arménie ne peut guère susciter d'ambitions commerciales de grande envergure. Quoi qu'il en soit, cet entrepreneur tente de convaincre ses partenaires arméniens de choisir l'Iran comme pays de transit pour les marchés asiatiques: "Avec ma femme, nous essayons d'aller voir des sociétés arméniennes et de les encourager de choisir l'Iran comme pays de transit pour leurs exportations avec la Chine, cela leur coûte moins cher de passer par l'Iran que par Batoumi. Parfois, on ramène des chocolats et des produits de consommation 'de luxe' en Iran." Et on ne peut pas dire que tout soit fait pour faciliter les échanges, vu la difficulté croissante d'obtenir le permis de résidence d'un an pour les ressortissants iraniens et la quasi-impossibilité d'obtenir la nationalité. En revanche, il est juridiquement possible d'exercer une activité entrepreneuriale en ne disposant que d'un visa de tourisme de quatre mois - ce qui contraint à une circulation régulière -, comme en ont témoigné des entrepreneurs familiaux que nous avons rencontrés.

\section{Le marché de l'automobile, un exemple des freins aux échanges}

Le marché de l'automobile permet de mieux saisir la place de l'Iran parmi les partenaires commerciaux de la région. Révélateur de l'imbrication entre acteurs publics et privés, informels et formels, il illustre surtout la manière dont fonctionnent le capitalisme d'initiés, la "pseudo-privatisation ${ }^{11}$ " et les monopoles sectoriels détenus par des personnes maitrisant les rouages administratifs et politiques, en guise de libéralisation en trompe-l'œil. Enfin, il prouve une fois de plus les effets pervers des sanctions internationales.

L'activité d'Iran Khodro en Arménie a commencé en 2000. En 2005, ses ventes, notamment des modèles Rana et Samand, étaient plutôt florissantes. Mais, avec le renforcement des sanctions internationales, la qualité de la production a commencé à baisser. Quand Peugeot a décidé d'arrêter de fournir des pièces aux usines d'Iran, les modèles ont cessé d'être compétitifs. Iran Khodro a donc dû remplacer les pièces produites par Peugeot par des pièces de moindre qualité venant de Chine, et les ventes ont baissé.

L'Arménie est, de plus, l'un des principaux pôles du marché d'exportation de véhicules d'occasion européen. Au lieu des 200 à 300 véhicules neufs escomptés par mois, Iran Khodro ne parvient à en vendre que 150 par an. La concurrence ne se fait pas entre voitures neuves, qu'elles viennent d'Europe ou d'Iran, mais entre occasions européennes 
celles des voitures Kia et Toyota, sont contrôlées par les instances de l'État qui privilégient ces compagnies : les licences de certaines grandes marques de voitures peuvent, selon des sources variées, être contrôlées par des représentants ou d'anciens représentants politiques. Cet exemple est éloquent des limites de la libéralisation des échanges : si certains accords sectoriels sont encadrés, d'autres le sont moins et révèlent l'imbrication entre sphère politique et sphère marchande, illustrant parfaitement le modèle "thermidorien" proposé par Jean-François Bayart ${ }^{12}$ d'une classe politique disposant des

La représentation d'Iran Khodro à Erevan. (c) Serge Weber.

et voitures neuves iraniennes, les Arméniens préférant acheter des européennes d'occasion. L'acheminement des voitures pose un autre problème à l'exportation iranienne : les occasions européennes passent par le port géorgien de Batoumi où les Arméniens se rendent pour acheter directement leur voiture, alors que les voitures iraniennes sont soumises aux conditions terrestres de transport. "On est obligé de prendre des camions transporteurs de six voitures et non de neuf, car les routes arméniennes ne sont pas adaptées." De plus, le tarif douanier arménien, qui est contrôlé par la Russie dans le cadre de l'EEU, pose des problèmes à la vente. Par exemple, un modèle vendu 7500 \$ en Iran est soumis à un droit de douane de $32 \%$ soit 2400 \$, auxquels s'ajoutent 700 \$ de transport, 100 \$ de débarquement et $1800 \$$ de TVA. Le prix de vente grimpe donc à 12500 \$ environ. Enfin, les pertes correspondant au change des devises ne sont compensées par des réductions de taxes qu'aux entreprises favorisées par des acteurs politiques locaux. Le principal obstacle est donc le monopole de l'État arménien sur les prix, qui ne contribue pas à fluidifier les échanges. D’autant plus que les principales licences,

\section{Conclusion}

À une heure d'avion, une journée d'autocar de Téhéran, Erevan fait partie de ce réseau régional de métropoles qui participent à l'accès, pour de nombreux Iraniens, à des formes de cosmopolitisme. Des villes comme Erevan constituent un espace familier pour faire partie du monde, échapper un temps aux contraintes économiques ou morales. Dans ces villes tremplins, que ce soit les clubs nocturnes, les boutiques, le choix des consommations, les stratégies de mise en ligne des photographies postées sur les réseaux sociaux, les rencontres, toutes ces expériences participent à une forme de subjectivation pour "devenir un homme (ou une femme) global(e)" (ensân-e-jahâni). Ces formes d'autonomisation individuelle, reposant sur des savoirfaire, des compétences et des négociations au jour le jour, développés dans des espaces étrangers certes, mais proches, participent aussi au rayonnement économique régional, formel et informel, de l'Iran. Le "tournevis français" est une figure emblématique de la professionnalisation des pratiques d'interface qui animent un "cosmopolitisme vernaculaire ${ }^{13}$ ". 